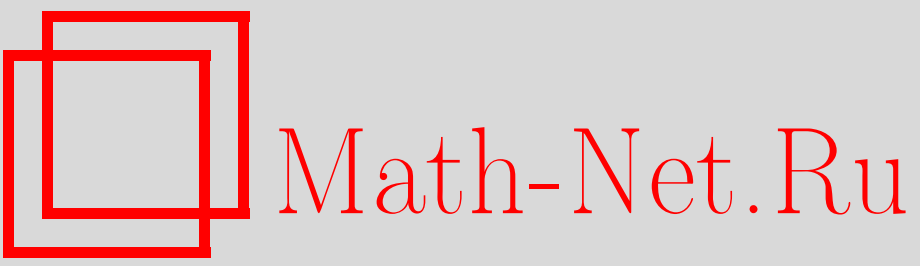

А. А. Лобашев, Н. Н. Трунов, Интегральный квазиклассический метод расчета спектров центрально-симметричных задач, ТMФ, 2000, том 124, номер 3, 463480

DOI: https://doi.org/10.4213/tmf651

Использование Общероссийского математического портала Math-Net.Ru подразумевает, что вы прочитали и согласны с пользовательским соглашением http: //www.mathnet.ru/rus/agreement

Параметры загрузки:

IP : 52.23 .180 .231

26 апреля 2023 г., $16: 30: 43$ 
ТЕОРЕТИЧЕСКАЯ

И МАТЕМАТИЧЕСКАЯ

ФИЗИКА

Том 124, № 3

сентябрь, 2000

(C) 2000 г.

А. А. Лобашев* ${ }^{*}$ Н.Н. Трунов ${ }^{\dagger}$

\section{ИНТЕГРАЛЬНЫЙ КВАЗИКЛАССИЧЕСКИЙ МЕТОД РАСЧЕТА СПЕКТРОВ ЦЕНТРАЛЬНО-СИММЕТРИЧНЫХ ЗАДАЧ}

Развит новый подход к квазиклассическим условиям квантования. Он основан на общих точных свойствах волновых уравнений и их спектров и не использует асимптотические разложения. Условия квантования для центрально-симметричных потенциалов зависят только от совокупности интегралов, включающих степени классического импульса. Значения уровней энергий, вычисленные с помощью этих условий, находятся в хорошем согласии с численными данными.

\section{1. ВВЕДЕНИЕ}

Отсутствие аналитических решений уравнения Шредингера для большинства потенциалов делает актуальным развитие приближенных методов для расчета спектров. Такие методы должны сочетать простоту и конструктивность с физической содержательностью. В первую очередь речь идет о центрально-симметричных потенциалах, имеющих широкое применение в физике атома, ядра, кваркония и т.д.

Важнейшим и наиболее универсальным из этих методов остается квазиклассический, который часто отождествляется с конкретной схемой реализации - ВКБ-представлением волновой функции в виде экспоненты с последуюшим асимптотическим разложением этой экспоненты по степеням малого параметра $\hbar$. При этом возникают фиктивные особенности в точках поворота, что заставляет дополнять метод сшивкой с точными решениями. Кроме того, поскольку фактическим параметром является $i \hbar \nabla$, метод ухудшает аналитические свойства волновой функции, включая в следуюших порядках многократные дифференцирования потенциала и предъявляя неоправданные требования к его гладкости. Это затрудняет оценки точности ведушего приближения, которым обычно и ограничиваются.

Между тем существует ряд точных результатов для спектров и решений волновых уравнений безотносительно к знанию аналитических решений. Сюда относятся возрас-

\footnotetext{
* Всероссийский научно-исследовательский институт метрологии им. Д.И. Менделеева, СанктПетербург, Россия. E-mail: lobashev@friedman.usr.lgu.spb.su

${ }^{\dagger}$ Всероссийский научно-исследовательский институт метрологии им. Д. И. Менделеева, СанктПетербург, Россия. E-mail: kukhar@onti.vniim.spb.su
} 
тание энергии уровней по мере роста одного из значений орбитального или радиального квантового числа при сохранении другого, асимптотически точные оценки для чисел связанных состояний в задачах разной размерности с данным потенциалом, своеобразные соотношения симметрии решений волновых уравнений для пар степенных потенциалов и т.д. Вышеупомянутая процедура оставляет возможность сколь угодно большого отклонения приближенных результатов от этих свойств.

Мы же предлагаем положить требование выполнения этих точных свойств в основу построения приближенного условия квантования. Наличие многих “опорных точек", к которым отнесем и совпадения с точными результатами для кулоновского и осцилляторного потенциалов, исключает значительные погрешности.

В данной статье применяется только главное приближение, игнорирующее градиенты потенциала; соответственно условие квантования включает интегрально зависящие от потенциала параметры - комбинации чисел состояний, отвечаюшие вышеуказанным условиям. При этом в рамках того же приближения по $ћ$ появляются внутренние критерии точности, зависящей от количества этих параметров и оптимальности их выбора.

Для центрально-симметричных задач уже простейшие одно- или двухпараметрические условия квантования при правильном выборе значений параметров дают высокую точность определения порядка уровней и спектров. Например, для степенных потенциалов правильно указывается порядок расщепления уровней при сколь угодно малом отклонении от осцилляторного потенциала и в то же время единообразно получаются достаточно точные значения уровней для всех значений показателей вплоть до бесконечного, которому соответствует неаналитический потенциал - прямоугольная яма.

Ниже частично используется аналитический аппарат [1], которому даются независимое обоснование и оценка точности. В отличие от работы [1], где изучалось появление новых состояний (с нулевой энергией) при увеличении амплитуды потенциала, в дальнейшем в нашей статье внимание сосредоточено на полном спектре при фиксированном потенциале. Обсуждаются возможное развитие метода квантования и его применение в других задачах вычислительной математики.

Используется атомная система единищ, часто встречающиеся числа $\nu, \lambda$ принимают положительные полуцелые значения.

\section{2. ПОЛУЧЕНИЕ ЛИНЕЙНОГО УСЛОВИЯ КВАНТОВАНИЯ}

Покажем, что как общий вид квазиклассического условия квантования, так и его линейный по орбитальному квантовому числу вариант для центрально-симметричных потенциалов могут быть получены на основе следуюших точных утверждений, известных или элементарно проверяемых.

2.1. Конкретизация обших выражений для асимптотических оценок числа $N_{p}$ состояний $[2, \S$ XIII.15] на случай центрально-симметричных потенциалов $V(r)=b v(r)$ с одной классически доступной областью дает для числа состояний с энергиями, не превышающими $E$, значение

$$
N_{p}(E, b \mid v)=\frac{M_{p}(E, b \mid v)}{2^{p-2} p^{2} \Gamma^{2}\left(\frac{p}{2}\right)},
$$


где

$$
M_{p}=\int \frac{d r}{r}\left[2 r^{2}(E-V(r))\right]^{\frac{p}{2}} .
$$

Здесь интегрирование идет между точками поворота, $p$ - размерность задачи, аргументы в $N_{p}, M_{p}$ (и далее в $\phi_{p}$ ) часто опускаем. Очевидно, что $N_{p}$ и $M_{p}$ можно употреблять на равных основаниях, причем $M_{p}$ определен и при нецелых значениях $p$.

Асимптотическое равенство

$$
\lim _{N_{p} \rightarrow \infty} \frac{N_{p}}{\widetilde{N}_{p}}=1,
$$

где $\tilde{N}_{p}$ - точное число состояний, фактически выполняется очень хорошо при малых $N_{p}$. Это, видимо, связано с тем, что исходные оценки справедливы при очень слабых условиях (отсутствие центральной симметрии и гладкости потенциала, наличие произвольного сочетания многих классически доступных и недоступных областей), причем метод получения оценок (вилка Дирихле-Неймана) не предполагает ВКБ-приближений. Мы же применяем оценки в благоприятном случае центрально-симметричного, гладкого потенциала с одной классически доступной областью и слабой (не сильнее кулоновской) сингулярностью в центре.

2.2. Уравнение Шредингера для широкого класса степенных потенциалов

$$
V(r)=b v(r), \quad v(r)=r^{\mu}
$$

c $-2<\mu<\infty, b \mu>0$, инвариантно относительно преобразования входящих в него параметров $(\lambda=l+1 / 2)$ :

$$
\left(\begin{array}{c}
E \\
b \\
\lambda \\
\mu
\end{array}\right) \rightarrow\left(\begin{array}{c}
E^{\prime}=-\sigma^{2} b \\
b^{\prime}=-\sigma^{2} E \\
\lambda^{\prime}=\sigma \lambda \\
\mu^{\prime}
\end{array}\right), \quad(2+\mu)\left(2+\mu^{\prime}\right)=4, \quad \sigma \equiv\left|\frac{\mu^{\prime}}{\mu}\right|
$$

при этом волновая функция умножается на положительную функцию [3].

Если $\mu>0$, то из (4) следует $-2<\mu^{\prime}<0$. В частности, воспроизводится известная сопряженность осцилляторного $\mu=2$ и кулоновского $\mu^{\prime}=-1$ потенциалов.

2.3. Ниже нам потребуются свойства $M_{p}(2)$ для конкретной функциональной формы $V(b, \lambda \mid v)=b v(r)+\lambda^{2} / r^{2}$ с постоянными $b, \lambda$; в явном виде можно записать, что

$$
M_{p}(E, b, \lambda \mid v)=\int d r r^{p-1}\left[2(E-b v(r))-\frac{\lambda^{2}}{r^{2}}\right]^{\frac{p}{2}} .
$$

Преобразование (4), примененное к $M_{p}(5)$, дает для степенных потенциалов (3)

$$
M_{p}\left(E^{\prime}, b^{\prime}, \lambda^{\prime} \mid \mu^{\prime}\right)=\sigma^{p-1} M_{p}(E, b, \lambda \mid \mu),
$$

где для краткости пишем аргумент $\mu$ вместо $r^{\mu}$. Учтем также однородность $M_{p}(E, b$, $\lambda \mid v)$ относительно аргументов, в частности

$$
M_{p}\left(a^{2} E, a^{2} b, a \lambda \mid \mu\right)=a^{p} M_{p}(E, b, \lambda \mid \mu)
$$

при любой $a=$ const $>0$. 
2.4. Потребуем, чтобы квазиклассическое условие квантования в степенных потенциалах (3) удовлетворяло точным соотношениям (4). Это с неизбежностью требует участия центробежного потенциала в виде $\lambda^{2} / r^{2}\left(\right.$ а не $\left.l(l+1) / r^{2}\right)$. Фактически мы имеем одномерную радиальную задачу с потенциалом $V(b, \lambda, r) \equiv V\left(b, \lambda \mid r^{\mu}\right)$; определяя для нее из (1) полное число состояний $N_{1}=M_{1} / \pi$, получим

$$
N_{1}(E, b, \lambda \mid \mu)=\frac{1}{\pi} M_{1}(E, b, \lambda \mid \mu)=n_{r}+C .
$$

Здесь $n_{r}=0,1,2, \ldots$ - радиальное квантовое число, а $C$ - поправка, учитывающая асимптотический характер $N_{1}$.

2.5. Условие (8) дает точный спектр для осциллятора и сопряженного ему кулоновского потенциала при $C=1 / 2$. Примем это значение $C$ для всех конечных $\mu>-2$. При $\mu=\infty$ получаем неаналитический потенциал - прямоугольную яму; учет непроницаемой стенки, так же как и в разделе 3 (см. ниже), приведет к известному значению $C=3 / 4$.

Для осциллятора

$$
M_{1}(E, b, \lambda \mid 2)=M_{1}(E, b, 0 \mid 2)+\pi \phi \lambda
$$

со значением фазы $\phi=1 / 2$ независимо от $E, b$. Для потенциалов $v \sim r^{\mu}$ с произвольными $\mu$ линейность $M_{1}$ по $\lambda$ выполняется при $\lambda \rightarrow 0$.

Согласно вышесказанному предположим, что линейность по $\lambda(9)$ сохраняется с достаточной точностью для всех потенциалов, тогда условие квантования (8) примет вид

$$
\frac{1}{\pi} M_{1}(E, b \mid v)=\nu+\phi(E, b) \lambda, \quad \nu=n_{r}+\frac{1}{2}
$$

Поставим целью выразить фазу ф через интегральные характеристики $M_{p} \equiv M_{p}(E$, $b, 0 \mid v)$ (согласно определениям (2), (5)). Для этого снова обратимся к степенным потенциалам. Инвариантность при преобразовании (4) значений $M_{1}$ (согласно (6) при $p=1)$, входящих в соотношение $(9)$, ведет и к инвариантности $\phi(\mu) \lambda=\phi\left(\mu^{\prime}\right) \lambda^{\prime}$. Учитьвая еше (7), получим требование к фазе

$$
\begin{aligned}
\sigma \phi\left(E^{\prime}, b^{\prime} \mid \mu^{\prime}\right) & =\phi(E, b \mid \mu), \\
\phi\left(a^{2} E, a^{2} b \mid \mu\right) & =\phi(E, b \mid \mu)=\phi(E / b, 1 \mid \mu) .
\end{aligned}
$$

Учитывая мультипликативный характер соотношений инвариантности (6), (7), (11), будем искать $\phi$ в виде

$$
\phi=C_{p, q} M_{p}^{\alpha} M_{q}^{\beta} \equiv \phi_{p, q}
$$

с неопределенными параметрами $\alpha, \beta, p, q, C_{p, q}$. Требование совместности $(6),(7),(11)$ приводит к уравнениям

$$
\alpha p+\beta q=0, \quad \alpha+\beta=1,
$$

оставляюшим произвольными две из четырех, входяших в (13), величин. 
Потребуем, чтобы для осциллятора было $\phi_{p, q} \equiv 1 / 2$ при всех $p, q$. Пользуясь явным выражением (2) для степенных потеншиалов, а также соотношениями (12) и (13), найдем для всех $\mu$

$$
\phi_{p, q}=\left[\frac{\left(\frac{M_{p}}{m_{p}}\right)^{q}}{\left(\frac{M_{q}}{m_{q}}\right)^{p}}\right]^{\frac{1}{q-p}}=\phi_{q, p}, \quad m_{p}=B\left(\frac{1}{2}, \frac{p}{2}\right),
$$

где $B$ - бета-функция (показатель $\mu$ входит в интегралы $M_{p}$ ).

Как будет видно из дальнейшего, целесообразно выбрать $p, q$ из пар чисел $(1,2)$ или $(1,3)$. Тогда мы придем к двум возможным значениям

$$
\phi=\phi_{1,2}=\frac{2}{\pi^{2}} \frac{M_{1}^{2}}{M_{2}}, \quad \phi=\phi_{1,3}=\frac{1}{\pi \sqrt{2}} \frac{M_{1}^{\frac{3}{2}}}{M_{3}^{\frac{1}{2}}},
$$

совпадаюшим для осциллятора. Для других значений $\mu$ величина

$$
R=\frac{\phi_{1,2}-\phi_{1,3}}{\phi_{1,3}}
$$

характеризует погрешность метода и оказывается малой (порядка $1 \div 2 \%$, см. далее раздел 4).

В потенциалах с неограниченным ростом чисел состояний, например кулоновском при $E \rightarrow 0$, растут и значения $\phi_{p}$ при каждом $p \neq 0$. Однако при этом остаются конечными их отношения $\phi_{p, q}(14)$, в частности $\phi_{1,2}, \phi_{1,3}$.

Далее мы распространяем условие (10) с фазами (15) на все потеншиалы. Для этого есть несколько аргументов. Во-первых, потенциал входит в $M_{p}$ и $\phi_{p}$ только функционально. Во-вторых, значения $R$ оказываются столь же малыми для всех реальных потенциалов. В-третьих, эти последние можно представить в виде степенных с медленно меняюшимся значением показателя (см. ниже (24)). Отметим, что формулы (15) совпадают с выражениями [1], полученными совершенно иным путем - сравнением чисел состояний, найденных разными способами.

Все вышеизложенное справедливо и для двумерной центрально-симметричной задачи; при том же потенциале $v$ единственное отличие - замена $\lambda$ на $|m|$, где $m$ - магнитное число. Паре состояний $\left(n_{r}, m\right)$ и $\left(n_{r},-m\right)$ соответствует в трехмерной задаче только один набор $\left(n_{r}, \lambda=|m|\right)$. Поэтому полное число состояний двумерной задачи $N_{2}$ из (1) вдвое больше количества оболочек трехмерной задачи: $N_{\mathrm{sh}}=N_{2} / 2$.

Теперь ясен физический смысл всех $M_{n}$ в условиях квантования и фазах: $N_{1}=$ $M_{1} / \pi$ (при любой размерности) определяет максимальное радиальное квантовое число, $N_{\mathrm{sh}}=M_{2} / 4$ - число оболочек, т.е. групп состояний с разными наборами $\left(n_{r}, l\right)$, $N_{3}=2 M_{3} / 3 \pi-$ полное число связанных состояний (все $M_{n}$ с энергиями состояний, не превышаюшими $E)$. Условие квантования (10), (15) использует минимальную информацию - одно значение $M_{n}$, кроме исходного $M_{1}$ в (10). 
В пренебрежении малой величиной $R$ можно отождествить $\phi_{1,2}$ и $\phi_{1,3}$ из (14), что позволяет выразить условие квантования через любую пару $M_{n}$. В частности, в форме

$$
N_{\text {sh }}=\left(\frac{9 \phi}{8}\right)^{\frac{1}{3}} N_{3}^{\frac{2}{3}}
$$

это условие непосредственно связывает число оболочек $N_{\mathrm{sh}}$ с полным числом связанных состояний в потенциале (с энергиями, не превосходящими $E$ ). Такая форма особенно удобна при анализе периодической системы элементов, где $N_{3}=Z / 2$ (спиновое удвоение даст атомный номер $Z$ ).

\section{3. КВАНТОВАНИЕ СФЕРИЧЕСКИ-СИММЕТРИЧНЫХ СОСТОЯНИЙ}

Отдельного рассмотрения заслуживает случай $l=0$, который может рассматриваться двояко: во-первых, как частный случай вышеизложенного при $\lambda=1 / 2$; во-вторых, как модифицированный одномерный: точный спектр $s$-состояний в потенциале $V(r)$ совпадает со спектром одномерной задачи на полуоси в потенциале $V_{1}(x)=V(x)$ при $x>0$ и $V_{1}(x)=\infty$ при $x=0$ (т.е. при обрашении волновой функции одномерной задачи в нуль при $x=0)$ [4, с. 91].

В нашем подходе по аналогии с изложенным в разделе 2 условие квантования должно принять вид

$$
\frac{1}{\pi} M_{1}=\frac{1}{\pi} \int \sqrt{2(E-V(r))} d r=n_{r}+c+g \phi_{p, q},
$$

где константы $c, g$ и параметры $p, q$ подлежат определению. Соотношения (4) теперь неприменимы (хотя бы потому, что содержат переменный параметр $\lambda$ ). Поскольку в трехмерной задаче физический смысл имели величины $M_{n}$ с $n \leqslant d=3$, естественно ожидать, что в одномерной задаче $\phi_{p, q}$ будет содержать $M_{n}$ с $n=0,1$. Как видно из (14), возможен только вариант, когда

$$
\phi_{01}=\lim _{p \rightarrow 0} \frac{M_{p}}{m_{p}}=\phi_{0},
$$

при этом $\phi_{1}^{0}=M_{1}^{0}=1$ выпадает. Величина $\phi_{0}$ из-за расходимости $M_{p}$ и $m_{p}$ определяется только асимптотическим поведением $M_{p}$ при $r \rightarrow 0$ и у (правой) точки поворота $r^{\prime}$, эта величина была вычислена в [1]. В задачах с асимптотикой $V \sim r^{\mu}$ при $r \rightarrow 0$ и стандартным линейным поведением $V(r)$ при $r \simeq r^{\prime}$ имеем

$$
\phi_{0}=\frac{1}{2} \quad(\mu \geqslant 0), \quad \phi_{0}=\frac{1}{2+\mu} \quad(\mu \leqslant 0)
$$

независимо от значения $E$. Требуя для кулоновского потенциала $\left(\phi_{0}=1\right)$ и осциллятора $\left(\phi_{0}=1 / 2\right)$ совпадения вычисленного по (17) $s$-спектра с точным, найдем $c=g=1 / 2$; при этом условие (17) совпадает с получаемыми из сшивки точных и ВКБ-решений $[5$, $\S 3,2]$ условиями. 
Как видно из предыдушего, соотношения (10) и (17) должны рассматриваться самостоятельно. Однако для удобства использования мы объединим условия (10) и (17), положив в (10)

$$
\phi=\phi_{0}, \quad l=0, \quad \phi=\phi_{1,3}, \quad l>0 .
$$

Очевидно, что для осциллятора и кулоновского потенциала $\phi_{0}=\phi_{1,3}$. В общем же случае наблюдается расхождение. Так, для степенных потенциалов $\phi_{0}=1 / 2$ независимо от $\mu>0$. В то же время $\phi_{1,3}$ пробегает при росте $\mu$ в интервале $(0, \infty)$ все значения в интервале $(0.643,0.390)$.

Для некоторых потенциалов могут быть найдены точные решения только для $s$-потенциалов (см. ниже раздел 5). Они подтверждают необходимость использования именно $\phi_{0}$, а не $\phi_{1,3}$ при $l=0$ (хотя в большинстве случаев при малой разности $\phi_{0}-\phi_{1,3}$ и при использовании $\phi_{1,3}$ получаются хорошие результаты, т.к. при этом в (17) возникает множитель $\lambda=1 / 2)$.

В заключение раздела сделаем несколько общих замечаний. Разрывньй характер (19) полностью соответствует физической ситуации: при сколь угодно малом $l>0$, pacсматриваемом как непрерывный параметр, одно из точных решений радиальной задачи становится сингулярным в нуле, а применению стандартного варианта метода ВКБ препятствует быстрое изменение де-бройлевской длины при малых $r$. Условие квантования, включающее $\lambda$ вместо исходного $l(l+1)$, в обычном подходе появляется как результат перехода к переменной $x=\ln r$ с одновременным преобразованием волновой функции, обеспечивающим отсутствие первой производной, получением ВКБ-условия для этой функции и возвратом к прежней переменной. Требование инвариантности (4) автоматически вводит в условие параметр $\lambda$ и тем самым выделяет переход к $r=\exp x$ среди всех возможных преобразований, обеспечивающих применимость метода ВКБ (в отличие от точного спектра, ВКБ-приближение неинвариантно, вообше говоря, относительно таких замен).

Отметим, наконец, что в некоторых задачах, например о появлении новых связанных состояний с энергией $E=0$ в атомах, точка поворота может уходить на бесконечность. Если $V \sim r^{-\alpha}, \alpha>2$ при $r \rightarrow \infty$, то при $E=0$ в $\phi_{0}$ (18) добавляется вклад $\phi_{>}=(\alpha-2)^{-1}$. Интегральные величины $\phi_{1,2}, \phi_{1,3}$ всегда являются плавными функциями энергии.

\section{4. ПРИМЕНЕНИЕ ЛИНЕЙНОГО УСЛОВИЯ}

Условие квантования, выраженное через $M_{p}(2)$, примет вид

$$
\frac{1}{\pi} M_{1}=\nu+\lambda \phi, \quad \nu \equiv n_{r}+\frac{1}{2},
$$

где в качестве $\phi$ фигурирует $\phi_{1,3}$ из (15).

Широкий класс потенциалов представляют степенные потенциалы $v=r^{\mu}(3)$, причем в силу п. 2.2 раздела 2 достаточно рассматривать $\mu>0$. В этом случае расчет $M_{p}(2)$ дает

$$
M_{p}=\frac{(2 E)^{\frac{p}{2}}}{\mu}\left(\frac{E}{b}\right)^{\frac{p}{\mu}} B\left(\frac{p}{\mu}, \frac{p}{2}+1\right) .
$$


Таблица 1

\begin{tabular}{|c|c|c|c|c|}
\hline$l$ & $n_{r}$ & $E^{(\mathrm{ex})}$ & $E^{(l)} / E^{(\mathrm{ex})}$ & $E^{(n)} / E^{(\mathrm{ex})}$ \\
\hline 0 & 0 & 1.8558 & 1.0115 & 1.0050 \\
0 & 1 & 3.2446 & 1.0068 & 1.0015 \\
0 & 2 & 4.3817 & 1.0047 & 1.0007 \\
1 & 0 & 2.6678 & 1.0039 & 1.0012 \\
1 & 1 & 3.8768 & 1.0072 & 1.0007 \\
1 & 2 & 4.9270 & 1.0068 & 1.0004 \\
2 & 0 & 3.3718 & 1.0003 & 1.0005 \\
2 & 1 & 4.4683 & 1.0057 & 1.0003 \\
10 & 0 & 7.5834 & 0.9937 & 1.0003 \\
10 & 1 & 8.3411 & 0.9980 & 1.0001 \\
\hline
\end{tabular}

Факторизация $E$ и $b$ в (21) приводит при всех $\mu$ к независимости фаз $\phi_{p, q}$ (в частности, $\phi_{1,2}$ и $\left.\phi_{1,3}\right)$ от энергии и амплитуды потенциала, что следует из соотношений симметрии раздела 2 .

Для уровней энергии $E^{(l)}(\nu, \lambda)$ в линейном по $\lambda$ приближении для всех степенных потенциалов (3) с $\mu>0$ получим

$$
E^{(l)}(\nu, \lambda)=b^{\frac{2}{2+\mu}}\left[(\nu+\phi \lambda) \frac{\mu \pi}{\sqrt{2} B\left(\frac{3}{2}, \frac{1}{\mu}\right)}\right]^{\frac{2 \mu}{2+\mu}}
$$

где

$$
\phi=\phi_{1,3}=\frac{1}{\mu \pi \sqrt{2}} \frac{B^{\frac{3}{2}}\left(\frac{1}{\mu}, \frac{3}{2}\right)}{B^{\frac{1}{2}\left(\frac{3}{\mu}, \frac{5}{2}\right)}} .
$$

В табл. 1 - 3 приведены сравнения вычисленных нашим методом и в результате численных расчетов [6] значений энергии для трех потенциалов. В табл. 1 приведены результаты для потенциала $V(r)=r$, даны отношения вычисленных спектров к точному значению $E^{(\mathrm{ex})}$ для линейного приближения $E^{(l)}(22)$ с $\phi_{1,3}=0.544$ и нелинейного приближения $E^{(n)}(51)$. В табл. 2 приведены результаты для потенциала $V(r)=r^{4} / 4$, даны отношения вычисленных спектров к точному значению $E^{(\mathrm{ex})}$ для линейного приближения $E^{(l)}(22)$ с $\phi_{1,3}=0.460$ и нелинейного приближения $E^{(n)}(51)$. В табл. 3 приведены результаты для потенциала прямоугольной ямы: $V(r)=0$ при $r \leqslant R, V(r)=\infty$ при $r>R$, даны отношения вычисленных амплитуд $A=R \sqrt{2 E}$ к точным, определяемым условием $J_{\lambda}\left(A^{(\mathrm{ex})}\right)=0$ для линейного приближения $A^{(l)}$ с $E^{(l)}(22)$ и $\phi_{1,3}=0.390$, для нелинейного приближения $A^{(n)}$ с $E^{(n)}(51)$. В данном случае $\nu=n_{r}+3 / 4$ (см. п. 2.5 раздела 2). Видно хорошее совпадение результатов. 
Таблица 2

\begin{tabular}{|c|c|c|c|c|}
\hline$l$ & $n_{r}$ & $E^{(\mathrm{ex})}$ & $E^{(l)} / E^{(\mathrm{ex})}$ & $E^{(n)} / E^{(\mathrm{ex})}$ \\
\hline 0 & 0 & 1.5079 & 0.9526 & 0.9715 \\
0 & 1 & 4.6212 & 0.9820 & 0.9947 \\
0 & 2 & 8.4285 & 0.9892 & 0.9982 \\
0 & 3 & 12.7383 & 0.9923 & 0.9992 \\
1 & 0 & 2.8210 & 0.9770 & 0.9900 \\
1 & 1 & 6.3626 & 0.9768 & 0.9962 \\
1 & 2 & 10.4570 & 0.9813 & 0.9984 \\
1 & 3 & 14.9907 & 0.9847 & 0.9993 \\
2 & 0 & 4.3028 & 0.9904 & 0.9953 \\
2 & 1 & 8.1924 & 0.9783 & 0.9970 \\
2 & 2 & 12.5463 & 0.9789 & 0.9983 \\
10 & 0 & 19.9638 & 1.0194 & 1.0014 \\
10 & 1 & 25.5651 & 1.0011 & 0.9999 \\
10 & 2 & 31.3926 & 0.9914 & 0.9993 \\
\hline
\end{tabular}

Величина $R(16)$, а с ней и относительная погрешность, очевидно, в классе $v=r^{\mu}$ растут вместе с отклонением $\mu$ от 2 ; однако даже в предельно неблагоприятном случае прямоугольной ямы $\mu \rightarrow \infty$, когда ухудшаются аналитические свойства $V$, величина $R$ не превышает $4 \%$ и метод дает хорошие результаты, обычно же $R \sim 1 \%$.

С помошью соотношений симметрии (4) или непосредственным расчетом $M_{p}$ получим аналогичные результаты для потенциалов атомного типа $-2<\mu^{\prime}<0$, имеюших нулевую асимптотику при $r \rightarrow \infty$ :

$$
\left|E^{(l)}(\nu, \lambda)\right|=|b|^{\frac{2}{2-\left|\mu^{\prime}\right|}}\left[\frac{1}{\nu+\phi \lambda} \frac{\sqrt{2} B\left(\frac{3}{2}, \frac{2-\left|\mu^{\prime}\right|}{2\left|\mu^{\prime}\right|}\right)}{\left|\mu^{\prime}\right| \pi}\right]^{\frac{2\left|\mu^{\prime}\right|}{2-\left|\mu^{\prime}\right|}},
$$

со значением фазы $\phi=\phi_{1,3}\left(\mu^{\prime}\right)$ для данного $\mu^{\prime}$. Непосредственно видно, что (23) и (22) переходят друг в друга при преобразовании (4). Как видно из (23), значение $E=0$ возможно лишь в пределе $\nu, \lambda \rightarrow \infty$, так что полное число уровней бесконечно. Изученная в [1] задача об определении параметров потенциала, при которых появляется очередной уровень с $E=0$, относилась к быстро убываюшим на бесконечности потенциалам (в первую очередь, экранированным кулоновским потенциалам).

Наш метод, основанный на величине $\phi_{1,3}$ как интегральной характеристике задачи, дает правильный порядок близких уровней с $n_{r}+l / 2=$ const, вырожденных для осциллятора ( $\mu=2)$. Известные методы ВКБ-анализа, приписывая (в наших обозначениях) 
Таблица 3

\begin{tabular}{|c|c|c|c|c|}
\hline$l$ & $n_{r}$ & $A^{(\mathrm{ex})}$ & $A^{(l)} / A^{(\mathrm{ex})}$ & $A^{(n)} / A^{(\mathrm{ex})}$ \\
\hline 0 & 0 & 3.1416 & 0.9449 & 0.9838 \\
0 & 1 & 6.2832 & 0.9725 & 0.9938 \\
1 & 0 & 4.4934 & 0.9332 & 0.9899 \\
1 & 1 & 7.7253 & 0.9495 & 0.9945 \\
2 & 0 & 5.7635 & 0.9401 & 0.9918 \\
2 & 1 & 9.0950 & 0.9411 & 0.9957 \\
3 & 0 & 6.9879 & 0.9506 & 0.9929 \\
3 & 1 & 10.4171 & 0.9393 & 0.9963 \\
5 & 0 & 9.3558 & 0.9718 & 0.9954 \\
5 & 1 & 12.9665 & 0.9435 & 0.9967 \\
7 & 0 & 11.6570 & 0.9901 & 0.9986 \\
7 & 1 & 15.4313 & 0.9515 & 0.9968 \\
10 & 0 & 15.0335 & 1.0121 & 1.0040 \\
10 & 1 & 19.0259 & 0.9649 & 0.9975 \\
\hline
\end{tabular}

всем регулярным в нуле потенциалам значение $\phi=\phi_{0}=1 / 2$, отождествляют эти уровни при всех $\mu[3,6]$. Как видно, отличие $\phi_{1,3}$ от $1 / 2$ получается в главном приближении (и не содержит параметра малости порядка $\hbar^{2}$ ).

Рассмотрим вопрос об изменении энергии уровня $\delta E$ и фазы $\delta \phi$ при малом изменении потенциала $\delta V$. Для степенных потенциалов $r^{\mu}$ и возмушений $\delta V=\alpha r^{\sigma}$, где $\mu, \sigma, \alpha>0$, вариация (20) выражается через бета-функции и приводит к $\delta \phi>0$ при $\sigma<\mu$. В то же время величина $\delta E>0$ в более широком интервале $\sigma<\sigma_{0}$, где граничное значение $\sigma_{0}$ удовлетворяет уравнению

$$
\frac{3 B\left(\frac{3}{2}, \frac{\sigma_{0}+3}{\mu}\right) B\left(\frac{3}{2}, \frac{1}{\mu}\right)}{B\left(\frac{5}{2}, \frac{3}{\mu}\right) B\left(\frac{1}{2}, \frac{\sigma_{0}+1}{\mu}\right)}=1 .
$$

Выполнение неравенств $\delta E>0$ при $\delta V>0$ утверждается теоремой Хелманна-Фейнмана [7]. За пределами интервала $\sigma<\sigma_{0}$, т.е. при слишком быстром возрастании $\max _{r} \delta V(r) / V(r)$ в классически доступной области при $E \rightarrow \infty$, линейное приближенное условие квантования (20) не обеспечивает выполнение теоремы Хелманна-Фейнмана. Нелинейное же условие раздела 6 удовлетворяет этой теореме при любых возмушениях.

Потенциал $V(r)$ общего вида приведет к некоторой зависимости фазы от энергии. Как правило, эта зависимость будет весьма медленной, т.к. типичный потенциал можно представить в виде степенного с показателем $k(V)$, медленно меняюшимся вместе с $V$ 
(монотонно, если эта зависимость физически отражает экранирование). Например, для всех экранированных кулоновских потенциалов $\phi \rightarrow 1$ для самого глубокого уровня, а при $E \rightarrow 0$ определяется данными таблицы из статьи [1]. Так, для потенциала Тайца $V=-\left(r\left[1+r^{2}\right]\right)^{-1}$ значение $\phi_{1,3} \rightarrow 2$ при $E \rightarrow 0$. Перейдем к функции $r(V)$ и монотонному $V(r)$. Основной вклад в $M_{n}$, а с ним и в $\phi_{1,3}$, даст окрестность $V \simeq V_{m}$, где $r^{2}\left(V_{m}\right)\left(E-V_{m}\right)=\max ($ ср. с выводом соотношений $(42),(43)$, полученных далее), т.е.

$$
E=V_{m}\left(1+\frac{k\left(V_{m}\right)}{2}\right), \quad k=\frac{r}{V}\left(\frac{d V}{d r}\right) .
$$

Для степенных потенциалов $k(V) \equiv \mu$, так что $k$ может рассматриваться как обобщение понятия показателя. В качестве примера рассмотрим потенциал

$$
V=\left(1+r^{2}\right)^{\frac{\mu}{2}}-1
$$

с асимптотиками $V \sim r^{2}$ при $r \rightarrow 0$ и $V \sim r^{\mu}$ при $r \rightarrow \infty$, считаем, что $\mu>2$. Из (24) имеем

$$
k(V)=\mu \frac{(1+V)^{1-\frac{2}{\mu}}}{V}\left[(1+V)^{\frac{2}{\mu}}-1\right],
$$

откуда $k(0)=2, k(\infty)=\mu$. Система $(24),(25)$ определяет $V_{m}(E)$; подставляя в $\phi_{1,3}(22)$ значение $\mu=k\left[V_{m}(E)\right]$, получим хорошее приближение для $\phi_{1,3}(E)$ с точными пределами при $E \rightarrow 0, \infty$.

\section{5. ИНФОРМАЦИЯ О СПЕКТРЕ}

Развитый выше метод позволяет получать информацию на трех различных уровнях, причем на каждом - в прямом и обратном вариантах.

5.1. Порядок следования уровней определяется непосредственно возрастанием эффективного квантового числа (равного правой части условия (20))

$$
T \equiv \nu+\phi \lambda \equiv\left(n_{r}+\frac{1}{2}\right)+\phi\left(l+\frac{1}{2}\right) \equiv T\left(n_{r}, l\right)
$$

по известной величине $\phi=\phi_{1,3}$ или с большей точностью по паре величин $\left(\phi_{0}, \phi_{1,3}(E)\right)$ (19). Обратно, знание реального порядка следования уровней позволяет получить границы для $\phi_{1,3}$ и $\phi_{0}$; однотипность этого порядка во всех областях спектра означает малую зависимость $\phi_{1,3}$ от энергии.

Аналогична задача о появлении новых уровней при фиксированной энергии $(E=0)$, но увеличении мошности потенциала $b$; она решалась в работах $[1,8]$.

5.2. Изучение локальных свойств спектра позволяет найти относительное энергетическое расстояние близлежаших уровней по $\phi(E)$ без использования $M_{1}$ из $(2)$ и тем самым без использования явного вида $V(r)$. Для последовательных уровней $1,2,3$ 
с энергиями $E$ и квантовыми числами $\nu, \lambda$, нумеруемыми теми же индексами, аппроксимируя зависимость $M_{1}(E)$ и $\phi(E)$ линейной зависимостью в интервале $\left(E_{1}, E_{3}\right)$, получим

$$
\begin{gathered}
D E_{12}=\nu_{12}+\phi\left(E_{2}\right) \lambda_{12}, \\
D E_{32}=\nu_{32}+\phi\left(E_{2}\right) \lambda_{32}, \\
D=\frac{1}{\pi} \frac{d M_{1}\left(E_{2}\right)}{d E}-\lambda_{2} \frac{d \phi\left(E_{2}\right)}{d E},
\end{gathered}
$$

где $E_{n m}=E_{n}-E_{m}$. Аналогичные соотношения можно записать для $\nu, \lambda$. Почленное деление двух уравнений системы (27) дает относительную разность интервалов $E_{12} / E_{13}$ как функцию только $\phi$ и квантовых чисел.

Обратно, при известных $E_{n m}, \lambda_{n m}, \nu_{n m}$ исключение из системы (27) величины $D$ позволяет определить

$$
\phi\left(E_{2}\right)=\frac{\nu_{32} E_{12}-\nu_{12} E_{32}}{\lambda_{12} E_{32}-\lambda_{32} E_{12}}
$$

при условии конечности знаменателя (вообе говоря, для этого достаточно, чтобы $\left.\lambda_{12}-\lambda_{32} \neq 0\right)$. Применение $(28)$ к последовательным тройкам уровней позволяет восстановить медленную функцию $\phi(E)$ (при необходимости с последующим сглаживани$\mathrm{eM})$.

В двухпараметрическом приближении $\left(\phi_{0}, \phi_{1,3}\right)$ необходимо исключить в $(28)$ состояния с $l=0$. Значение $\phi_{0}$ (не зависящее от энергии) получим аналогично (27):

$$
\phi_{0}=2 \frac{E_{32}\left(\lambda_{1} \phi+\nu_{12}\right)-E_{12}\left(\lambda_{3} \phi+\nu_{32}\right)}{E_{3}-E_{1}} .
$$

Здесь предполагается, что $s$-уровнем является только средний уровень с энергией $E_{2}$ и номером $\nu_{2}$, к этой же энергии относится $\phi=\phi_{1,3}$.

5.3. Наконец, зная $V(r)$, можно определить $M_{1}, \phi_{0}, \phi_{1,3}$ и найти спектр в иелом.

Развитый метод позволяет восстановить весь спектр, если для данного потенциала известны решения при $l=0$. Из вида условия квантования (10) следует, что энергия уровня $E$ и потенциал $V=b v(r)$ фиксируют зависимость $E$ от $b$ и $\nu, \lambda$ с точностью до функции $f$, определяемой видом $v(r)$ :

$$
f\left(\frac{E_{n_{r} l}}{b}\right)=\frac{T\left(n_{r}, l\right)}{\sqrt{2 b}}, \quad E_{n_{r} l}=b F\left(\frac{T\left(n_{r}, l\right)}{\sqrt{2 b}}\right) .
$$

Здесь $F$ - функция, обратная $f$, число $T\left(n_{r}, l\right)$ берется из $(26)$. Оно же входит в выражение для энергий уровней (22), (23).

Сравним (30) с видом точных решений, известных для потенциалов

$$
\begin{aligned}
& V=\frac{b}{\operatorname{ch}^{2} r} \\
& V=-\frac{b}{e^{r}-1}
\end{aligned}
$$


только при $l=0$ и $n_{r}=0,1, \ldots$. Имеем

$$
\begin{array}{ll}
E_{n_{r} 0}=-b\left(\sqrt{1-\frac{1}{8 b}}-2 u\right)^{2}, & u \equiv \frac{n_{r}+\frac{3}{4}}{\sqrt{2 b}}, \\
E_{n_{r} 0}=-\frac{b\left(1-u^{2}\right)^{2}}{2 u^{2}}, & u \equiv \frac{n_{r}+1}{\sqrt{2 b}} .
\end{array}
$$

Выражение (32а) получим как нечетные состояния в одномерном потенциале [4, с. 60], (32б) взято из работы [9]. Обрашает на себя внимание в формуле (32а) множитель $\sqrt{1-1 /(8 b)}$, нарушаюший функциональную зависимость (30), в то время как вид (32б) точно соответствует (30). Следует ожидать поэтому, что для формулы (32б) точные и ВКБ-решения совпадают при $l=0$, а для $(32 \mathrm{a})$ различаются заменой радикала на единищу; это действительно имеет место.

Далее, из (32) следует, что потенциалам (31) при $l=0$ (т.е. $\lambda=1 / 2$ ) действительно нужно приписать фазы

$$
\begin{aligned}
& \phi=\phi_{0}=\frac{1}{2}, \\
& \phi=\phi_{0}=1,
\end{aligned}
$$

отличные от фаз $\phi_{1,2}$ и $\phi_{1,3}$.

Явные формулы типа (32) позволяют распространить результаты на спектр при всех $l \neq 0$. Для этого необходимо выполнить в (32) замену

$$
n_{r} \rightarrow n_{r}+\frac{1}{2}+\left(l+\frac{1}{2}\right) \phi_{1,3}-\frac{\phi_{0}}{2}
$$

\section{6. НЕЛИНЕЙНОЕ УСЛОВИЕ КВАНТОВАНИЯ}

Для повышения точности линейных условий учтем возможную нелинейность $M_{1}$ из (8):

$$
M_{1}(\lambda)=M_{1}(0)-A \Delta(\Lambda), \quad \Delta(\Lambda)=\int_{0}^{\Lambda} \phi(\Lambda) d \Lambda,
$$

где опущены несущественные аргументы и обозначено

$$
A^{2}=\max _{r} \widetilde{W}(E, r), \quad \widetilde{W}(E, r)=2 r^{2}(E-V(r)), \quad 0 \leqslant \Lambda=\frac{\lambda}{A} \leqslant 1
$$

$\Lambda$ рассматривается как непрерывная переменная в промежуточных вычислениях; при $\phi(\Lambda)=$ const возврашаемся к линейным соотношениям $(9),(10)$. Обратим внимание на то, что в данном разделе объектом изучения является именно обобшенный потенциал $\widetilde{W}(36)$, а не исходный потенциал $V(r)$. Меллиновский образ $\phi_{p} / p$ функции $\phi(\Lambda)$ просто связан с $M_{p}[1]$ соотношением

$$
\phi_{p}=p \int_{0}^{1} \phi(\Lambda) \Lambda^{p-1} d \Lambda=\frac{M_{p}}{A^{p} m_{p}}
$$


с тем же $m_{p}$, что и в (14). Справедливы следующие общие свойства:

$$
\phi_{0}=\phi(\Lambda=0), \quad \phi_{\infty}=\phi(\Lambda=1) .
$$

Для изученных потенциалов функции $\phi_{p}$ и $\phi(\Lambda)$ являются монотонными. В частности, для степенных потенциалов $(3)(\mu>0)$ имеем

$$
\phi_{p}=\frac{(\mu+2)^{\frac{p}{\mu}+\frac{p}{2}}}{2^{\frac{p}{\mu}} \mu^{\frac{p}{2}+1}} \frac{B\left(\frac{p}{\mu}, \frac{p}{2}+1\right)}{B\left(\frac{p}{2}, \frac{1}{2}\right)} .
$$

Ранее введенные фазы $\phi_{p, q}(14)$ могут быть выражены через $\phi_{p}(37)$ с помощью формулы

$$
\phi_{p, q}^{q-p}=\frac{\phi_{p}^{q}}{\phi_{q}^{p}},
$$

причем множители, пропоршиональные $A^{p q}$, выпадают.

Информацию о качественном поведении $\phi_{p}$ будем извлекать из фактической близости $\phi_{1,2}$ и $\phi_{1,3}$ и инвариантности $M_{p}$ относительно допустимых деформаций $\widetilde{W}[1]$, сохраняющих $\phi_{p}$ и $\phi(\Lambda)$. С учетом (40) имеем

$$
\left(\frac{\phi_{1,2}}{\phi_{1,3}}\right)^{2}=\frac{\phi_{1} \phi_{3}}{\phi_{2}^{2}}=\frac{8}{\pi^{2}} \frac{M_{1} M_{3}}{M_{2}^{2}} \simeq 1
$$

с малой погрешностью, не большей чем $2 R$. Универсальность выполнения условия (41) указывает на утрату в $M_{p}$ при $p \geqslant 1$ индивидуальных особенностей $\widetilde{W}(r)$, что характерно для асимптотического поведения. Действительно, $M_{n}$ тождественны интегралам, асимптотика которых получена Лапласом,

$$
M_{p}=\int d x W^{\frac{p}{2}}(x) \sim \sqrt{\frac{4 \pi}{\left|W^{(2)}\right| p}} A^{p+1}\left(1+\frac{a_{1}}{p}+\frac{a_{2}}{p^{2}}+\cdots\right) .
$$

Здесь выполнена замена переменной $x=\ln r$, так что $W(x)=\widetilde{W}(\exp x)$; все производные $W^{(n)}$ берутся в точке максимума. Если подставить в (41) только главные члены разложения, заменяя скобку в (42) единищей, то получим $16 /\left(\pi^{2} \sqrt{3}\right)$, что менее чем на $4 \%$ отличается от единицы. Это подтверждает предположение об асимптотическом поведении $\phi_{p} \sim M_{p}$ при $p \geqslant 1$.

Заменой $W=\exp S$ интеграл (42) приводится к стандартной форме; в коэффициентах разложения, приведенных в книге [10], необходимо затем вернуться к $W$. В итоге все коэффишиенты $a_{n}$ выражаются через комбинации производных, инвариантные относительно масштабирования $W \rightarrow F W, x \rightarrow c x$. В частности,

$$
a_{1}=\frac{i_{1}(W)-3}{4}, \quad i_{1}=A^{2}\left(\frac{W^{(4)}}{\left[W^{(2)}\right]^{2}}-\frac{5}{3} \frac{\left[W^{(3)}\right]^{2}}{\left[W^{(2)}\right]^{3}}\right)
$$


Вьполняя разложение $m_{p}$ в знаменателе (37), аналогичное (42), получим асимптотическое разложение $\phi_{p}$, которое удобнее записать в экспоненциальной форме (что возможно и в $(42))$

$$
\frac{\phi_{p}}{\phi_{\infty}}=\exp \left[\frac{b_{1}}{p}+\frac{b_{2}}{p^{2}}+\cdots\right], \quad \phi_{\infty}=A \sqrt{\frac{2}{\left|W^{(2)}\right|}}, \quad b_{1}=\frac{i_{1}}{4}-1 .
$$

Выражение для $\phi_{\infty}$ совпадает с полученным в [1] иным способом.

Быстрому выходу $\phi_{p}$ на асимптотику соответствует $b_{1}<1, b_{2} \ll b_{1}$ (особенно при $\left.b_{1} b_{2}<0\right)$ и т.д. Такое положение имеет место для $\Gamma$-функций, определяюших $M_{n}$ для степенных и многих других потенциалов: отношение $\Gamma(p)$ к стирлинговской асимптотике $\Gamma_{0}(p)$ имеет вид экспоненты (44) с $b_{1}=1 / 12, b_{2}=-1 / 360$. Конечная причина малости характерного параметра $p^{*}<1$, с которого начинается асимптотика, состоит в следующем: $\Gamma(p)$ может быть представлена в виде [11]

$$
\frac{d^{2}}{d p^{2}} \ln \Gamma(p)=\sum_{n=0}^{\infty} \frac{1}{(p+n)^{2}}=I_{1}(p)+I_{2}(p)
$$

причем $I_{1}$ является заменой суммы интегралом по $d n$ и дает как раз $\Gamma_{0}$. Поправка к $\Gamma_{0}$ определяется интегралом $I_{2}$ метода Абеля-Плана с экспоненциальным знаменателем и характерным для $I_{2}$ параметром затухания $p^{*} \sim(2 \pi)^{-1} \simeq 0.2$ (см. примеры в [12]).

Отметим, что через произведение гамма-функций или отношение таких произведений выражаются интегралы от чрезвычайно большого числа функций с качественно различным поведением (их таблицы занимают более 150 страниц монографии [13]). Соответственно обсужденные вьшше свойства асимптотик можно считать типичными.

Справедливость предположения об асимптотике вида (44) с быстро убываюшими $b_{n}$ подтверждается и следующим образом. Ограничиваясь членами $b_{1}, b_{2}$, найдем по (16) и (40)

$$
R=\frac{\phi_{1,2}}{\phi_{1,3}}-1=\exp \left(\frac{1}{6} b_{1}+\frac{11}{36} b_{2}\right)-1 .
$$

Предполагая $\left|b_{2}\right| \ll\left|b_{1}\right|$, получим для потенциалов из таблицы статьи [1] при учете $|R| \sim 1 \div 2 \%$ оценку $b_{1} \leqslant 0.1$. Подбирая комбинацию $\phi_{p}$ так, чтобы обратить в нуль коэффициент при $b_{1}$, например $\phi_{1} \phi_{\infty} / \phi_{2}^{2}$, мы уменьшим отклонение от единишы, как правило, до $10^{-3}$. Наконец, обрашение в нуль и коэффишиента при $b_{2}$, например

$$
\frac{\phi_{1}^{1 / 3} \phi_{3}^{3}}{\phi_{2}^{8 / 3} \phi_{\infty}^{2 / 3}} \equiv 1+R^{\prime}
$$

уменьшает ошибку $R^{\prime}$ до величины порядка $10^{-4}$.

Асимптотика (44) правильно описывает замедление изменения $\phi_{p}$ при $p \geqslant 1$. Если ограничиться только членом с $b_{1}$, то из условия $\phi_{0}=\phi_{\infty} \exp \left(b_{1} / p^{\prime}\right)$ можно оценить для потенциалов таблицы из [1] нижнюю границу $p^{\prime} \sim 0.2 \div 0.3$, до которой ведущую роль играет $b_{1}$. 
В обшем случае ряд по обратным степеням $p$ функции $\phi_{p} / p$, являющейся преобразованием Меллина для $\phi(\Lambda)$, определяет эту функцию при всех $\Lambda$, а поэтому и $\phi_{p}$ при всех $p$ вплоть до $p=0$. В силу конечности $\phi(\Lambda=0)=\phi_{0}$ это означает, что ряд в экспоненте (44) должен сворачиваться в регулярную при $p=0$ функцию $p$; это обстоятельство необходимо учитьвать в приближенных представлениях $\phi_{p}$.

Наконец, асимптотика (44) объясняет крайнюю близость фазы $\phi_{1,3}$ к среднеквадратичной фазе

$$
\bar{\phi}=\frac{3 \phi_{1}-\phi_{3}}{2} .
$$

Напомним, что в статье [1] было введено понятие расстояния между эффективными потенциалами $W_{1,2}$, определяемое разностью найденных для них $\Delta_{1,2}$ из (35):

$$
\rho\left(W_{1}, W_{2}\right)=\int_{0}^{1}\left[\Delta_{1}(\Lambda)-\Delta_{2}(\Lambda)\right]^{2} .
$$

Фаза $\bar{\phi}$ получена как наилучшее приближение заданной функции $\Delta_{1}(\Lambda)$ линейным значением $\Delta_{2}=\bar{\phi} \Lambda$, минимизируюшим $\rho$. В отношении $\bar{\phi}$ и $\phi_{1,3}=\left(\phi_{1}^{3} / \phi_{3}\right)^{1 / 2}$ выпадает линейньй по $b_{1}$ член, что и приводит к совпадению указанных величин с точностью порядка $10^{-3}$. При сравнении $\bar{\phi}$ с $\phi_{1,2}$ линейное по $b_{1}$ расхождение сохраняется, однако $\phi_{1,2}$ по той же причине близко к величине $2 \phi_{1}-\phi_{2}$, получаемой минимизацией $\rho$ с дополнительным весом $d \Lambda \rightarrow d \Lambda / \Lambda$. Такая минимизация актуальна в задачах с преимушественным значением малых орбитальных моментов.

Вышеприведенные оценки базируются на асимптотике (42), предполагающей наличие требуемых производных $W^{(n)}$. Однако, как упоминалось выше, погрешность метода и, в частности, разность фаз $\bar{\phi}-\phi_{1,3}$ лишь незначительно возрастает для неаналитических потенциалов, что является одним из преимушеств интегрального подхода.

В [1] были изучены допустимые деформации эффективного потенциала $W(36)$ при неизменной $A$, сохраняющие функции $\phi(\Lambda), \phi_{p}$ и $M_{p}$. При этом с неизбежностью требуется выразить коэффициенты рядов $(42),(44)$ через инварианты таких деформаций. Простейшим подобным выражением является $i_{0}=W^{(2)}$. Следующий коэффициент $i_{1}(43)$, как нетрудно получить, одинаков для осциллятора $W=e^{2 x}\left(1-e^{2 x}\right)$ и потенциала Ленца $W=\operatorname{ch}^{-2}(2 x)$, при этом в обоих случаях $\phi(\Lambda)=1 / 2$. Обратно, исходя из требований инвариантности, сведения ряда (42) к первому члену при $W=e^{-x^{2}}$ и постоянства $\phi_{p}$ для осциллятора, можно было бы восстановить ряд (44). Инварианты $i_{n}$ последовательно определяются условием сохранения при деформации ширины потенциальной ямы $\left|x_{-}(W)\right|+x_{+}(W)$ на каждом уровне $W$ для двух склонов $x_{-}<0, x_{+}>0$. При этом функция $W$ заменяется возрастаюшими по длине отрезками ряда Тейлора.

\section{7. ПРИМЕНЕНИЕ НЕЛИНЕЙНОГО УСЛОВИЯ КВАНТОВАНИЯ}

Для состояний с моментом $l \neq 0$ и произвольной функции $\phi(\Lambda)$ из (8) и $(35)$ следует условие, обобщающее (20),

$$
\frac{1}{\pi} M_{1}=\nu+A \int_{0}^{\frac{\lambda}{A}} \phi(\Lambda) d \Lambda
$$


с амплитудой $A$ из (36).

Физически значимая информация о $\phi_{p}(37)$ содержится в основном в фазах $\phi_{n} \sim N_{n}$ с $n=1,2,3$, определяюших числа состояний, и в факте конечности $\phi_{p}$ при $p \rightarrow 0, \infty$. $\mathrm{C}$ практически достаточной точностью поведение $\phi_{p}$ хорошо приближается дробно-линейной зависимостью с тремя параметрами. Если принять условие совпадения точных значений $\phi_{n} \sim M_{n}$ с вычисленными по приближенной формуле для $n=1,2,3$, то получим [1] следующее универсальное выражение:

$$
\Delta(\Lambda)=\int_{0}^{\Lambda} \phi(\Lambda) d \Lambda=(\chi+\kappa) \Lambda-\frac{\kappa}{\tau+1} \Lambda^{\tau+1},
$$

где

$$
\begin{gathered}
\tau+1=\frac{2\left(\phi_{2}-\phi_{3}\right)}{\phi_{1}+\phi_{3}-2 \phi_{2}}, \\
\kappa=2 \frac{\left(\phi_{2}-\phi_{1}\right)\left(\phi_{1}-\phi_{3}\right)\left(\phi_{3}-\phi_{2}\right)}{\left(4 \phi_{2}-3 \phi_{3}-\phi_{1}\right)\left(\phi_{1}+\phi_{3}-2 \phi_{2}\right)}, \\
\chi=\phi_{1}+\frac{\left(\phi_{2}-\phi_{1}\right)\left(\phi_{1}-\phi_{3}\right)}{\left(\phi_{1}+\phi_{3}-2 \phi_{2}\right)} .
\end{gathered}
$$

В случае степенных потеншиалов (3) величины $\phi_{p}$ не будут зависеть от $E$ или $b$, так что в условии квантования (48) эти параметры появятся лишь через функцию $A$,

$$
A(E)=\left(\frac{2 \mu E}{\mu+2}\right)^{\frac{\mu+2}{2 \mu}} \text { при } b=\frac{1}{\mu} .
$$

Для энергий уровней $E^{(n)}(\nu, \lambda)$ в нелинейном приближении

$$
E^{(n)}(\nu, \lambda)=b^{\frac{2}{2+\mu}}\left[\left(\nu+(\chi+\kappa) \lambda-\frac{\kappa}{\tau+1}\left(\frac{\lambda}{A(E)}\right)^{\tau} \lambda\right) \frac{\mu \pi}{\sqrt{2} B\left(\frac{3}{2}, \frac{1}{\mu}\right)}\right]^{\frac{2 \mu}{2+\mu}}
$$

имеем алгебраическое уравнение, поскольку $E$ посредством функции $A(E)(50)$ входит и в правую часть. Уравнение (51) можно решать с помощью итераций. Для этого подставим в выражение (51) амплитуду $A\left(E^{(l)}\right)(50)$, вычисленную на энергиях $E^{(l)}(\nu, \lambda)$ линейного приближения (22). Таким образом, для энергий уровней всех степенных потенциалов (3) в нелинейном приближении получаем явное, хотя и несколько громоздкое, выражение. Результаты вычислений энергий уровней по этой явной итерационной формуле (при частных значениях $\mu$ ) в сравнении с результатами численных расчетов [6] приведены в табл. $1-3$.

Точными решениями для прямоугольной сферической ямы являются, как известно, функции Бесселя полуцелого значка $J_{\lambda}(z)$. Обычные асимптотики для них имеют разный вид в зависимости от соотношения $\lambda$ и $z$. В нашем же подходе получаем равномерное приближение корней функций Бесселя при любом отношении $\lambda / z$, причем, очевидно, числу $\lambda$ можно придавать и другие (не полуцелые) значения. 
Данный потенциал примечателен и своими плохими аналитическими свойствами, что отражается в значении $\phi_{\infty}=0$. Тем не менее значения корней определяются достаточно точно.

Таким образом, развитый подход обеспечивает простое и конструктивное определение спектра центрально-симметричных потенциалов. При решении задач каждого типа используется минимально необходимый объем информации, что полностью соответствует методу сокращенного описания [1].

Предложенный метод имеет много возможностей развития и применения. Среди них назовем, например, учет следуюшего по $\hbar^{2}$ приближения [1]. Отдельного изучения заслуживает обратная задача о восстановлении потенциала с учетом допустимых его деформаций [1].

Намеченная выше связь задачи с методом Лапласа позволяет предложить интерполяционные аналоги этого метода для неаналитических функций, определяемые только интегральными характеристиками.

Наконец, изучение асимптотических свойств фаз дает способ получения нелинейных соотношений между специальными функциями, выполняемых с большой точностью. Таково, например, соотношение (46) для степенных потенциалов, выражаемое через бета-функции при использовании явного вида $\phi_{p}(39)$.

\section{Список литературы}

[1] А. А. Лобашев, Н. Н. Трунов. ТМФ. 1999. Т. 120. С. 99.

[2] М. Рид, Б. Саймон. Анализ операторов. Методы современной математической физики. Т. 4. М.: Мир, 1982.

[3] C. Quigg, J. L. Rosner. Phys. Rep. 1979. V. 56. P. 167.

[4] П. В. Елютин, В. Д. Кривченков. Квантовая механика с задачами. М.: Наука, 1976.

[5] А. Б. Мигдал. Качественные методы квантовой теории. М.: Наука, 1974.

[6] Б. М. Карнаков, В. Д. Мур, В. С. Попов. ЖЭТФ. 1995. Т. 107. С. 1768.

[7] A.А. Локшин, Е.А. Сагомонян. Геометрические методы в теории спектров. М.: Изд-во МГУ, 1996.

[8] Ю.В. Тарбеев, Н.Н. Трунов, А.А. Лобашев, В.В. Кухарь. ЖЭТФ. 1997. Т. 112. C. 1226 .

[9] З. Флюгحе. Задачи по квантовой механике. Т. 1. М.: Мир, 1974.

[10] М. В. Федорюк. Асимптотика: интегралы и ряды. М.: Наука, 1987.

[11] Э. Т. Уиттекер, Дж. Н. Ватсон. Курс современного анализа. Ч. II. М.: Физматгиз, 1963.

[12] В. М. Мостепаненко, Н. Н. Трунов. Эффект Казимира и его приложения. М.: Энергоатомиздат, 1990.

[13] О.И. Маричев. Метод вычисления интегралов от специальных функций (теория и таблицы формул). Минск: Наука и техника, 1978.

Поступила в редакцию 17.I.2000 г., после доработки 9.III.2000 г. 